\title{
MESH FREE ESTIMATION OF THE STRUCTURE MODEL INDEX
}

\author{
JOACHIM OHSER ${ }^{1}$, Claudia REDENBACH${ }^{2}$ AND KATJA SCHLADitz ${ }^{3}$ \\ ${ }^{1}$ Hochschule Darmstadt, Fachbereich Mathematik und Naturwissenschaften, Schöfferstraße 3, D-64295 \\ Darmstadt, Germany; ${ }^{2}$ Technische Universität Kaiserslautern, Fachbereich Mathematik, \\ Erwin-Schrödinger-Straße, D-67653 Kaiserslautern, Germany; ${ }^{3}$ Fraunhofer-Institut für Techno- und \\ Wirtschaftsmathematik, Abteilung Bildverarbeitung, Fraunhofer-Platz 1, D-67663 Kaiserslautern, Germany. \\ e-mail: jo@h-da.de, redenbach@mathematik.uni-kl.de,katja.schladitz@itwm.fraunhofer.de \\ (Accepted September 30, 2009)
}

\begin{abstract}
The structure model index (SMI) is a means of subsuming the topology of a homogeneous random closed set under just one number, similar to the isoperimetric shape factors used for compact sets. Originally, the SMI is defined as a function of volume fraction, specific surface area and first derivative of the specific surface area, where the derivative is defined and computed using a surface meshing. The generalised Steiner formula yields however a derivative of the specific surface area that is - up to a constant - the density of the integral of mean curvature. Consequently, an SMI can be defined without referring to a discretisation and it can be estimated from $3 \mathrm{D}$ image data without need to mesh the surface but using the number of occurrences of $2 \times 2 \times 2$ pixel configurations, only. Obviously, it is impossible to completely describe a random closed set by one number. In this paper, Boolean models of balls and infinite straight cylinders serve as cautionary examples pointing out the limitations of the SMI. Nevertheless, shape factors like the SMI can be valuable tools for comparing similar structures. This is illustrated on real microstructures of ice, foams, and paper.
\end{abstract}

Keywords: image analysis, integral of mean curvature, intrinsic volume densities, random closed set, shape factor.

\section{INTRODUCTION}

Nowadays, a variety of imaging techniques - first of all computed tomography, but also so-called FIB tomography, electron tomography or atomic force microscopy - are able to produce high quality 3D images of microstructures, increasing the demand for subsequent quantitative analysis. Assuming macroscopic homogeneity, the microstructure can be modelled by a stationary (or macroscopically homogeneous) random closed set.

In many applications, geometric characteristics of the random closed set have to be estimated from the given image. A very attractive set of such global geometric characteristics are the densities of the intrinsic volumes (or quermassintegrals or Minkowski functionals). In 3D, they are, up to constants, volume fraction $V_{V}$, surface area density $S_{V}$, density of the integral of mean curvature $M_{V}$, and Euler number density $\chi_{V}$.

These characteristics can be estimated efficiently from observations in digital binary (black-and-white) images based on discretised Crofton intersection formulae (Ohser et al., 2009; Ohser and Schladitz, 2009). These classical integral geometric formulae allow to compute the intrinsic volumes by calculation of Euler numbers in lower dimensional intersections and subsequent integration over all positions of the intersecting affine subspaces. The Euler numbers in turn can be determined efficiently using the EulerPoincaré formula for all $2 \times 2 \times 2$ pixel configurations and exploiting additivity. The core of the algorithm first outlined by Lang et al. (2001) consists in a convolution of the binary image with a $2 \times 2 \times 2$ mask, resulting in an 8 bit grey value image, coding the $2 \times 2 \times 2$ pixel configurations in the original binary image. Subsequently, the grey value histogram of this image is multiplied by a vector of suitable weights to derive the desired intrinsic volume. It is particularly noteworthy that the size of the grey value histogram does not depend on image size or content. Moreover all measurements are deduced directly from the pixel configurations there is no need to approximate the surface by a surface mesh.

A further characteristic for macroscopically homogeneous random sets $\Xi$ in the Euclidean space $\mathbb{R}^{3}$ is the structure model index (SMI) defined as

$$
f_{\mathrm{SMI}}=6 \frac{V_{V} S_{V}^{\prime}}{S_{V}^{2}}
$$

where $S_{V}^{\prime}$ denotes the 'first derivative' of the surface density $S_{V}$. The SMI was first suggested by Hildebrand and Rüegsegger (1997a;b) for evaluating bone structure.

In order to define the derivative $S_{V}^{\prime}$, Hildebrand and Rüegsegger use a surface meshing. Roughly, the mesh 
is moved outwards slightly thus defining a dilation of $\Xi$ by a small ball. The derivative is then approximated by the difference quotient. In this paper, $S_{V}^{\prime}$ is defined via the Steiner formula. As a consequence, the SMI can be expressed in terms of the densities of the intrinsic volumes which in turn allows to estimate the SMI from the grey value histogram of the convolved image described above. The definition for the SMI without referring to a discretisation as well as the mesh free estimator are derived in the section below.

Being a shape factor, the SMI can clearly not capture completely the random closed set under consideration. This is emphasised by the possible ranges for the SMI of Boolean models of balls and infinite straight cylinders. On the other hand, the SMI is helpful for comparing structures that are sufficiently similar like the pore systems in Greenland firn from different depths or for assessing the degree of closedness of technical foams.

\section{DERIVATION OF THE MESH FREE ESTIMATOR}

The class of all compact convex sets (convex bodies) in $\mathbb{R}^{3}$ is denoted by $\mathscr{K}$. Furthermore, we use the symbol $\mathscr{R}$ for the convex ring consisting of all finite unions of convex bodies. Finally, we introduce the extended convex ring $\mathscr{S}$ consisting of all sets $X \subset \mathbb{R}^{3}$ such that $X \cap K$ is an element of $\mathscr{R}$ for each $K \in \mathscr{K}$. Denote by $B_{r}$ a ball with radius $r$ and centred in the origin. Let $B(x, r)$ be a ball with centre $x$ and radius $r$, that is $B(x, r)=B_{r}+x$.

The Steiner formula expresses the volume of the parallel set $K \oplus B_{r}$ of a convex body $K$ at distance $r>0$ as a polynomial of the intrinsic volumes of $K$ and $B_{r}$,

$$
V\left(K \oplus B_{r}\right)=\sum_{k=0}^{3} r^{3-k} \kappa_{3-k} V_{k}(K), \quad r \geq 0, K \in \mathscr{K},
$$

(Schneider, 1993, p. 197). Here $\kappa_{k}$ denotes the volume of the $k$-dimensional unit ball. The intrinsic volumes $V_{k}, k=0, \ldots, 3$, are defined by (1). They are up to constants volume $V=V_{3}$, surface area $S=2 V_{2}$, integral of mean curvature $M=\pi V_{1}$, and Euler number $\chi=V_{0}$, and the integral of mean curvature is closely related to the mean width $M=2 \pi \bar{b}$.

The Steiner formula can be extended to the convex ring $\mathscr{R}$. We use Schneider's index function $j(X, x, y)$ of a set $X \in \mathscr{R}$ at $x$ with respect to $y$, as defined in Schneider and Weil (2008, Section 14.4). Using the
Euler number $\chi$, the index $j$ is defined as

$$
j(X, x, y)= \begin{cases}\lim _{\delta \downarrow 0} \lim _{\varepsilon \downarrow 0} \chi(X \cap B(x, \delta) \cap B(y,\|x-y\|-\varepsilon)), & \text { if } x \in X, \\ 0, & \text { otherwise }\end{cases}
$$

for all $X \in \mathscr{R}$ and $x, y \in \mathbb{R}^{3}$. It follows from the additivity of the Euler number that the index $j$ is additive in its first argument, too. Now, introducing local parallel sets with multiplicity we define the local measure $\rho_{r}(X, \cdot)$ by

$$
\begin{aligned}
\rho_{r}(X, A) & =\int_{\mathbb{R}^{3}} c_{r}(X, A, y) d y, \quad r>0 \\
\text { with } c_{r}(X, A, y) & =\sum_{x \in A \backslash\{y\}} j(X \cap B(y, r), x, y),
\end{aligned}
$$

for Borel sets $A \subseteq \mathbb{R}^{3}$. Here the sum is taken over only finitely many summands different from zero. The functional $\rho_{r}$ inherits the additivity from the index $j$. The Steiner formula for the local functional $\rho_{r}$ and its extension on the convex ring is given in Schneider (1993, Section 4.4) and Schneider and Weil (2008, Section 14.4). Here we will use the special case $A=$ $\mathbb{R}^{3}$, only. That is, we consider the functional

$$
\bar{\rho}_{r}(X)=\rho_{r}\left(X, \mathbb{R}^{3}\right) .
$$

Let now $\Xi$ be a macroscopically homogeneous random closed set on $\mathbb{R}^{3}$ with realisations of $\Xi$ almost surely belonging to the extended convex ring $\mathscr{S}$. We assume that $\Xi$ is observed through a compact and convex window $W$ with nonempty interior. Moreover, assume that $\Xi$ fulfils the integrability condition $\mathbb{E} 2^{\#(\Xi \cap K)}<\infty$ for any convex body $K \in \mathscr{K}$, where $\# X$ denotes the minimal number $m$ such that the set $X$ has a representation $X=K_{1} \cup \ldots \cup K_{m}$ with $K_{1}, \ldots, K_{m} \in \mathscr{K}$.

The volume density $V_{V, 3}$ of $\Xi$ is the expectation of the volume fraction of $\Xi$ in $W$,

$$
V_{V, 3}(\Xi)=\frac{\mathbb{E} V_{3}(\Xi \cap W)}{V(W)},
$$

$V(W)>0$. This definition of the volume density can be extended to the densities of the other intrinsic volumes. The realisations of $\Xi$ intersected with $a W, a>0$ are poly-convex sets. Hence, the intrinsic volumes $V_{k}(\Xi \cap a W), k=0, \ldots, 3$, exist and the intrinsic volume densities $V_{V, k}$ of $\Xi$ can be defined by the limits

$$
V_{V, k}(\Xi)=\lim _{a \rightarrow \infty} \frac{\mathbb{E} V_{k}(\Xi \cap a W)}{V(a W)}, \quad k=0,1,2,
$$

(cf. Schneider and Weil, 2008). In 3D the intrinsic volume densities are (up to multiplicative constants) the volume density $V_{V}=V_{V, 3}$, the surface density or specific surface area $S_{V}=2 V_{V, 2}$, the density of the integral of mean curvature $M_{V}=\pi V_{V, 1}$, and the density of the Euler number $\chi_{V}=V_{V, 0}$. 
The functional $\bar{\rho}_{r}(X)$ as defined in Equation (2) is additive, translation invariant and locally bounded and, hence, its density

$$
\bar{\rho}_{V, r}(\Xi)=\lim _{a \rightarrow \infty} \frac{\mathbb{E} \bar{\rho}_{r}(\Xi \cap a W)}{V(a W)},
$$

exists and satisfies a Steiner-type formula, too, as $\bar{\rho}_{r}$ does:

$$
\bar{\rho}_{V, r}(\Xi)=\sum_{k=0}^{3} r^{3-k} \kappa_{3-k} V_{V, k}(\Xi), \quad r \geq 0,
$$

(see Schneider and Weil, 2008, p. 428). Now it follows that

$$
\left[\frac{\mathrm{d}}{\mathrm{d} r} \bar{\rho}_{V, r}(\Xi)\right]_{r=0}=\kappa_{1} V_{V, 2}(\Xi)=S_{V}
$$

and

$$
\left[\frac{\mathrm{d}^{2}}{\mathrm{~d} r^{2}} \bar{\rho}_{V, r}(\Xi)\right]_{r=0}=2 \kappa_{2} V_{V, 1}(\Xi)=2 M_{V}
$$

In this sense we formally write $S_{V}^{\prime}=2 M_{V}$ and the structure model index $f_{\mathrm{SMI}}$ is given by

$$
f_{\mathrm{SMI}}=12 \frac{V_{V} M_{V}}{S_{V}^{2}}
$$

which has a similar structure as a shape factor for convex bodies: Consider the three isoperimetric shape factors for $K \in \mathscr{K}$

$$
\begin{aligned}
& f_{1}(K)=6 \sqrt{\pi} \frac{V(K)}{\sqrt{S^{3}(K)}}, \quad f_{2}(K)=48 \pi^{2} \frac{V(K)}{M^{3}(K)}, \\
& f_{3}(K)=4 \pi \frac{S(K)}{M^{2}(K)} .
\end{aligned}
$$

These shape factors are normalised such that $f_{1}\left(B_{r}\right)=f_{2}\left(B_{r}\right)=f_{3}\left(B_{r}\right)=1$. Deviations from 1 describe various aspects of deviations from ball shape. The shape factor $f_{4}(K)=f_{2}(K) /\left(3 f_{3}^{2}(K)\right)=$ $3 V(K) M(K) / S^{2}(K)$ derived from $f_{2}$ and $f_{3}$ is analogous to the structure model index which thus can be seen as a shape factor for random sets.

Note however, that $S_{V}^{\prime}$ in this sense is in general not the same as the derivative of $S_{V}$ derived from an infintesimal dilation as defined by Hildebrand and Rüegsegger (1997a). Roughly speaking, this is due to the fact that the index function used in the definition of the functional $\rho$ counts signed surface points and thus does not describe a dilation in the case of overlapping grains. Nevertheless, the $f_{\mathrm{SMI}}$ as defined here coincides with the orignal SMI by Hildebrand and Rüegsegger (1997a) for non-overlapping grains.
For an example where the two concepts differ see the following section.

For a system $\Xi$ of non-overlapping balls of constant radius $r$ with ball density (mean number of balls per volume unit) $\lambda$ we get

$$
f_{\mathrm{SMI}}(\Xi)=12 \frac{V_{V}(\Xi) M_{V}(\Xi)}{S_{V}^{2}(\Xi)}=12 \frac{\lambda \frac{4}{3} \pi r^{3} \lambda 2 \pi 2 r}{\lambda^{2} 16 \pi^{2} r^{4}}=4 .
$$

A macroscopically homogeneous system of platelike structures can only be obtained as a dilated random hyperplane system. Thus we have $M_{V}=0$ and consequently $f_{\mathrm{SMI}}=0$, too.

The third special case considered by Hildebrand and Rüegsegger (1997a;b) are spherical cylinders. Let $\Xi$ be a random system of non-overlapping infinite spherical cylinders of constant radius $r$, that is a random system of dilated straight lines. This can be achieved e.g. by dilating a system of lines parallel to the $\mathrm{z}$-axis whose feet form a hard core point process in the $x-y$-plane. Let $\Xi$ have length density (mean total length per volume unit) $\lambda$. Then $V_{V}(\Xi)=\lambda \bar{A}=\lambda \pi r^{2}$, $S_{V}(\Xi)=\lambda \bar{L}=\lambda 2 \pi r$, and $M_{V}(\Xi)=\lambda \pi$, where $\bar{A}$ and $\bar{L}$ denote the section area and the section circumference of the cylinders. Thus

$$
f_{\mathrm{SMI}}(\Xi)=12 \frac{\lambda \pi r^{2} \lambda \pi}{(\lambda 2 \pi r)^{2}}=3 .
$$

\section{SMI FOR BOOLEAN MODELS}

Let $\Xi$ be a homogeneous and isotropic Boolean model in $\mathbb{R}^{3}$ with typical grain $X_{0}$ and density $\lambda$. That is, $\Xi=\bigcup_{i=1}^{\infty}\left(x_{i}+X_{i}\right)$, where $\Phi=\left\{x_{i}\right\}_{i=1}^{\infty}$ is a homogeneous Poisson point field, the $X_{i}$ are i. i. d. like $X_{0}$, isotropic, and independent of $\Phi$. Let $\bar{V}, \bar{S}, \overline{\bar{b}}$ denote the expectations of the volume, the surface area, and the mean width of the grain $X_{0}$, respectively, i.e., $\bar{V}=\mathbb{E} V_{3}\left(X_{0}\right), \bar{S}=2 \mathbb{E} V_{2}\left(X_{0}\right)$ and $\overline{\bar{b}}=\frac{1}{2} \mathbb{E} V_{1}\left(X_{0}\right)$. Then the volume fraction $V_{V}$, the surface density $S_{V}$, and the density of the integral of the mean curvature $M_{V}$ of $\Xi$ can be expressed in terms of $\lambda, \bar{V}, \bar{S}$, and $\overline{\bar{b}}$ by Miles' formulae:

$$
\begin{aligned}
V_{V}(\Xi) & =1-\mathrm{e}^{-\lambda \bar{V}}, \\
S_{V}(\Xi) & =\mathrm{e}^{-\lambda \bar{V}} \lambda \bar{S}, \\
M_{V}(\Xi) & =\mathrm{e}^{-\lambda \bar{V}}\left(2 \pi \lambda \overline{\bar{b}}-\frac{\pi^{2} \lambda^{2}}{32} \bar{S}^{2}\right),
\end{aligned}
$$

(Miles, 1976), which is a special case of Schneider and Weil (2008, p. 389). By definition of the SMI we get 


$$
\begin{aligned}
& f_{\text {SMI }}(\Xi)= 12\left(1-\mathrm{e}^{-\lambda \bar{V}}\right) \mathrm{e}^{-\lambda \bar{V}}\left(2 \pi \lambda \overline{\bar{b}}-\frac{\pi^{2} \lambda^{2}}{32} \bar{S}^{2}\right) \\
& \cdot \mathrm{e}^{2 \lambda \bar{V}} \frac{1}{\lambda^{2} \bar{S}^{2}} \\
&=24 \pi\left(\mathrm{e}^{\lambda \bar{V}}-1\right)\left(\frac{\overline{\bar{b}}}{\lambda \bar{S}^{2}}-\frac{\pi}{64}\right) .
\end{aligned}
$$

The dilation of a Boolean model is the same as the Boolean model of the dilated grains ( $c f$. Chadœf et al., 2008). Thus a derivative of $S_{V}$ can also be deduced from Miles' formula. As already noted in the previous section, this is not the same as $S_{V}^{\prime}$ defined above. Consider the special case of the typical grain $X_{0}=B_{r}$ being a ball of constant radius $r>0$. We have

$$
S_{V}(\Xi)=\mathrm{e}^{-\lambda \frac{4}{3} \pi r^{3}} 4 \pi \lambda r^{2}
$$

and thus

$$
S_{V}(\Xi)^{\prime}=\mathrm{e}^{-\lambda \frac{4}{3} \pi r^{3}}\left(8 \pi \lambda r-\left(4 \pi \lambda r^{2}\right)^{2}\right)
$$

while

$$
2 M_{V}(\Xi)=\mathrm{e}^{-\lambda \frac{4}{3} \pi r^{3}}\left(8 \pi \lambda r-\left(\pi^{2} \lambda r^{2}\right)^{2}\right) .
$$

Nevertheless, all following considerations regarding the significance of $f_{\text {SMI }}$ hold analogously for the SMI in the sense of Hildebrand and Rüegsegger (1997a), too.

In the special case of the typical grain $X_{0}=B_{r}$ being a ball of constant radius $r>0$ this further simplifies to

$$
f_{\mathrm{SMI}}(\Xi)=3\left(\mathrm{e}^{\lambda \frac{4}{3} \pi r^{3}}-1\right)\left(\frac{1}{\lambda \pi r^{3}}-\frac{\pi^{2}}{8}\right) .
$$

Thus $\lim _{\lambda \rightarrow \infty} f_{\mathrm{SMI}}(\Xi)=-\infty$. On the other hand, $f_{\text {SMI }}(\Xi)>0$ for $\lambda<8 / \pi^{3} r^{3}$. Hence, already for one model - the Boolean model with constant ball radius $f_{\text {SMI }}$ can attain a wide range of values.

As a second example we consider now a Boolean cylinder model formed by a Poisson line field with each line dilated by a convex body $K \in \mathscr{K}$. The union of the resulting cylinders is a (generalised) Boolean model. More precisely, let $\Phi=\left\{L_{i}\right\}_{i=1}^{\infty}$ be a macroscopically homogeneous and isotropic Poisson point field in the space of straight lines in $\mathbb{R}^{3}$. Let $K_{1}, K_{2}, \ldots$ be i. i. d. convex bodies. Then the random closed set $\Xi=\bigcup_{i=1}^{\infty}\left(L_{i} \oplus K_{i}\right)$ is a Boolean model of infinite straight cylinders.

In the special case of the $K_{i}=B_{r_{i}}$ being balls, formulae for the densities of the intrinsic volumes in terms of the model parameters were derived by Davy (1978), see also Ohser and Schladitz (2009). Let the $r_{i}$ be i. i. d. as $r_{0}$ and denote by $\bar{A}=\pi \mathbb{E} r_{0}^{2}$ the mean cylinder section area and $\bar{L}=2 \pi \mathbb{E} r_{0}$ the mean circumference. The density of the Poisson line field is $\lambda$. The Miles' formulae for $V_{V}, S_{V}$ and $M_{V}$ now become

$$
\begin{aligned}
V_{V} & =1-\mathrm{e}^{-\lambda \bar{A}} \\
S_{V} & =\lambda \bar{L} \mathrm{e}^{-\lambda \bar{A}} \\
M_{V} & =\left(\pi \lambda-\frac{\pi^{2}}{32}(\lambda \bar{L})^{2}\right) \mathrm{e}^{-\lambda \bar{A}} .
\end{aligned}
$$

Spiess and Spodarev (2009) proved the surface density formula for the anisotropic case and general $K_{i}$. Hoffmann (2007a;b) derived further generalisations to inhomogeneous Poisson line fields.

In the case of the typical grain $K_{i}=B_{r}$ being a ball of constant radius $r>0$ the Miles' formulae further simplify to

$$
\begin{aligned}
V_{V} & =1-\mathrm{e}^{-\lambda \pi r^{2}} \\
S_{V} & =\mathrm{e}^{-\lambda \pi r^{2}} 2 \pi \lambda r \\
M_{V} & =\mathrm{e}^{-\lambda \pi r^{2}} \pi \lambda\left(1-\frac{\pi^{3}}{8} \lambda r^{2}\right),
\end{aligned}
$$

which gives for the SMI

$$
\begin{aligned}
f_{\text {SMI }}(\Xi) & =12 \frac{\left(1-\mathrm{e}^{-\lambda \pi r^{2}}\right) \pi \lambda\left(1-\frac{\pi^{3}}{8} \lambda r^{2}\right) \mathrm{e}^{-\lambda \pi r^{2}}}{4 \pi^{2} \lambda^{2} r^{2}\left(\mathrm{e}^{-\lambda \pi r^{2}}\right)^{2}} \\
& =3\left(\mathrm{e}^{\lambda \pi r^{2}}-1\right)\left(\frac{1}{\lambda \pi r^{2}}-\frac{\pi^{2}}{8}\right) .
\end{aligned}
$$

Thus, $\lim _{\lambda \rightarrow \infty} f_{\mathrm{SMI}}(\Xi)=-\infty$ while $f_{\mathrm{SMI}}(\Xi)>0$ for $\lambda<8 / \pi^{3} r^{2}$.

Given the ranges for the SMI for Boolean models of balls and infinite straight cylinders derived above, there are clearly sets of parameters such that the systems of overlapping balls and the cylinder system have the same SMI. As a special case consider Boolean models $\Xi_{b}$ of balls with point density $\lambda_{b}$ and fixed ball radius $r_{b}$ and $\Xi_{c}$ of cylinders with length density $\lambda_{c}$ and cross section radius $r_{c}$. Then $f_{\mathrm{SMI}}\left(\Xi_{b}\right)=f_{\mathrm{SMI}}\left(\Xi_{c}\right)=0$ if

$$
\lambda_{b}=\frac{8}{\pi^{3} r_{b}^{3}} \quad \text { and } \quad \lambda_{c}=\frac{8}{\pi^{3} r_{c}^{2}}
$$

These equations hold for instance for $\lambda_{b}=1000, r_{b}=$ $0.064, \lambda_{c}=286.68$, and $r_{c}=0.03$. Realisations of these two models, which are obviously not plate-like, are shown in Fig. 1. 


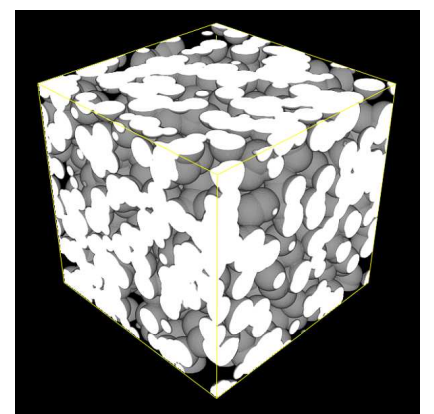

(a)

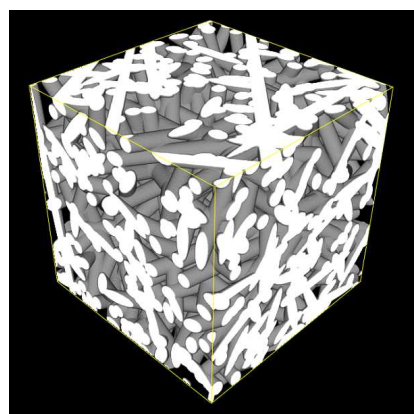

(b)
Fig. 1. Visualisations of realisations of Boolean models of spheres and cylinders with theoretical value $f_{\mathrm{SMI}}=$ 0. (a) Boolean model of spheres, estimated $\widehat{f}_{\mathrm{SMI}}\left(\Xi_{b}\right)=$ -0.022 , (b) Boolean model of cylinders, estimated $\widehat{f}_{\mathrm{SMI}}\left(\Xi_{c}\right)=-0.200$.

\section{APPLICATION}

The section above shows that the informative value of $f_{\mathrm{SMI}}$ is rather restricted. This holds however for all shape factors. Nevertheless, $f_{\mathrm{SMI}}$ is surely a valuable tool for comparing similar microstructures. In the following we will discuss several examples.

\section{GREENLAND FIRN (SINTERED SNOW)}

During the densification of polar firn, significant changes of the microstructure can be observed ( $c f$. Freitag et al., 2004, and references therein). These include a decrease of porosity with increasing depth but also changes of the topological structure of the pore space from a connected system of pore channels to a system of isolated spherical air bubbles. The SMI may be used as a means to characterise these changes.

As an example we analysed several samples of firn from the firn core B26 which was drilled during the North Greenland traverse of the Alfred Wegener Institute Bremerhaven in 1995. The borehole was located at $77^{\circ} 15^{\prime} \mathrm{N}, 49^{\circ} 13^{\prime} \mathrm{W}$. Five firn samples taken from different depths within the ice core were imaged using a portable $\mu \mathrm{CT}$ scanner (1074SR SkyScan) inside a cold room at $-25^{\circ} \mathrm{C}$. The analysis is based on grey value images consisting of $400^{3}$ pixels with a pixel size of $40 \mu \mathrm{m}$. From these, binary images of the pore system of the firn were obtained by global thresholding by Freitag et al. (2004). Visualisations of the firn samples are shown in Fig. 2.

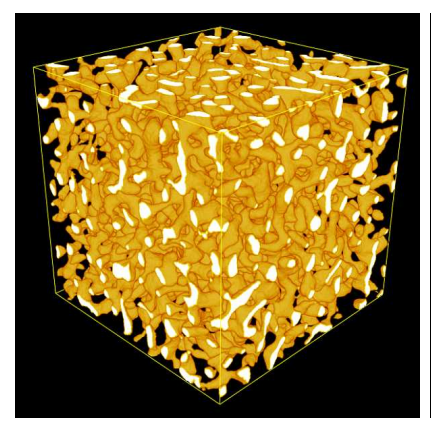

(a) $56 \mathrm{~m}$

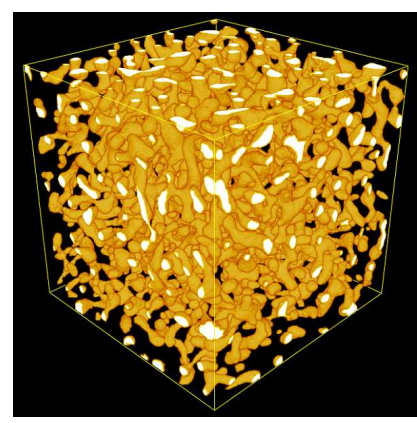

(c) $69 \mathrm{~m}$

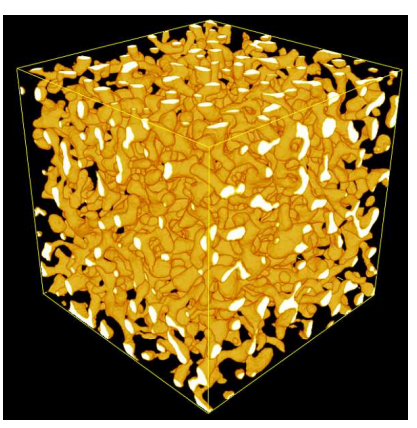

(b) $60 \mathrm{~m}$

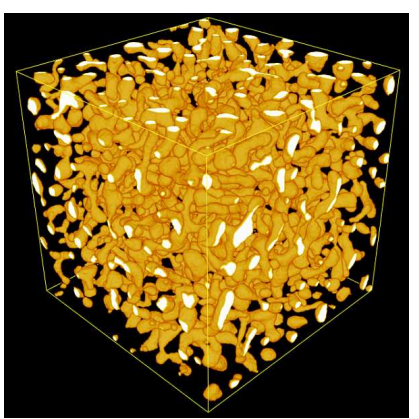

(d) $72 \mathrm{~m}$

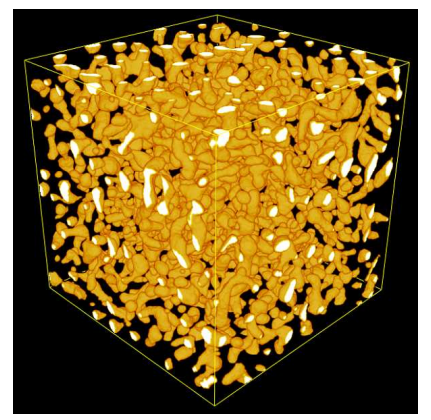

(e) $74 \mathrm{~m}$

Fig. 2. Visualisations of reconstructed tomographic images of firn samples from five different depths. Samples and imaging: J. Freitag, AlfredWegener Institute for Polar and Marine Research, Bremerhaven. The pore system is visualised.

The estimated values given in Table 1 show an increase of $f_{\text {SMI }}$ with increasing depth. The starting value around 3 indicates a cylindrical structure. The visualisations of the deeper samples already show a number of isolated spherical pores. Therefore, a further increase of $f_{\text {SMI }}$ towards 4 can be expected when going deeper within the firn core.

Table 1. SMI for firn samples with different porosities.

\begin{tabular}{lcc}
\hline depth $[\mathrm{m}]$ & porosity $[\%]$ & $\widehat{f}_{\text {SMI }}$ \\
\hline 56 & 15.71 & 2.954 \\
60 & 13.16 & 3.135 \\
69 & 10.11 & 3.373 \\
72 & 9.06 & 3.410 \\
74 & 7.83 & 3.503 \\
\hline
\end{tabular}




\section{TECHNICAL FOAMS}

Foams are used in an increasing number of application areas including filters, heat exchangers or sound absorbers. They are divided into open-cell foams consisting of a connected system of struts and closed-cell foams whose cells are bounded by membrane-like walls. However, also mixed forms with varying proportions of closed walls can be observed. The degree of closedness of a foam plays an important role for its macroscopic properties. Therefore, easy ways for its characterisation are highly desirable. Here, we propose the SMI as a measure for the closedness of a foam.

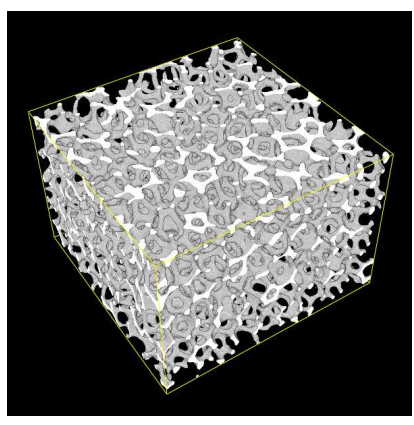

(a)

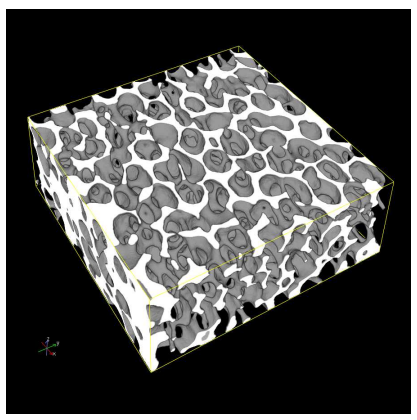

(c)

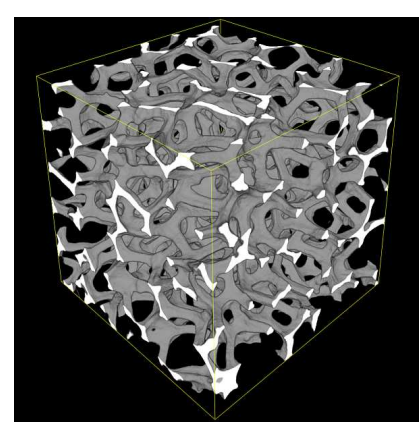

(b)

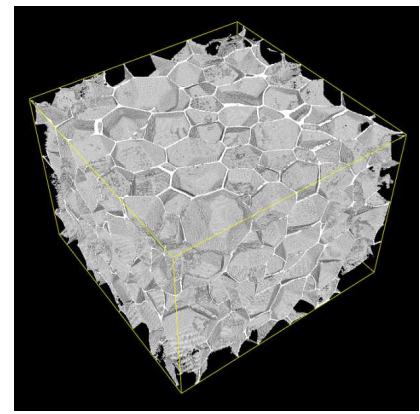

(d)
Fig. 3. Visualisations of reconstructed tomographic images of four foam samples. (a) Open aluminium foam, sample: m-pore GmbH, imaging: Fraunhofer IZFP, (b) Open nickel-chromium foam, sample: Recemat Int. (RCM-NC-2733.10), imaging: RJL Micro \& Analytic, (c) Partly open ceramic foam, sample: FOSECO GmbH, imaging: Fraunhofer IZFP, (d) Closed polymer foam, sample and imaging: $R$. Schlimper, Fraunhofer IWM.

We estimated the SMI from tomographic images of the following foam samples showing different proportions of closed walls:

- an open aluminium foam, $820 \times 820 \times 278$ pixels, pixel size $64.57 \mu \mathrm{m}$,

- a nickel-chromium foam, used as sound absorber, $800 \times 1600 \times 1600$ pixels, pixel size $3.14 \mu \mathrm{m}$,
- a ceramic foam, used for filtering metal melts, $670 \times 670 \times 270$ pixels, pixel size $70.88 \mu \mathrm{m}$,

- a closed polymethacrylimide (PMI) foam, used as lightweight core material for sandwich applications, $480 \times 480 \times 360$ pixels, pixel size $10.21 \mu \mathrm{m}$.

Visualisations of the tomographic images of the foam samples are shown in Fig. 3. The estimated SMIs are given in Table 2. None of the estimated SMIs is near the value for an ideal cylinder structure. However, the two open foams have a significantly higher SMI than the (partially) closed samples and the SMI of the closed PMI foam is close to the expected value 0 . Moreover, the difference between the strut shape between the aluminium foam (round) and the nickelchromium foam (trilobal) is clearly reflected by the SMI, too.

Table 2. SMI for the foam samples.

\begin{tabular}{ll}
\hline sample & $\widehat{f}_{\text {SMI }}$ \\
\hline aluminium & 2.188 \\
nickel-chromium & 1.695 \\
ceramic & 0.429 \\
polymer & 0.207 \\
\hline
\end{tabular}

\section{PAPER}

The microstructure of paper determines important properties like tensile strength or filtration properties. Therefore, it has been studied for a long time, however mainly based on $2 \mathrm{~d}$ images. Here we use a 3D image of a recycling paper sample obtained by synchrotron-based phase contrast microtomography at beamline ID22 of the European light source ESRF (employing an effective pixel size of $0.7 \mu \mathrm{m}$ corresponding to approx. $2 \mu \mathrm{m}$ spatial resolution, $13 \mathrm{keV}$ monochromatic photon energy). For further details on the experimental setup and the phase retrieval algorithm applied see Weitkamp et al. (1999) and Paganin et al. (2002), respectively. Most of the cellulose fibres are collapsed and the paper contains various additives. This causes the microstructure to be very irregular. Nevertheless, estimation of the SMI on seven $260^{3}$ pixel subvolumes yields the values 0.19 , $0.37,0.50,-0.08,0.2,0.14,0.29$, still indicating a rather plate like structure.

Fig. 4 shows visualisations of the two samples with the minimal and maximal estimated SMI. 


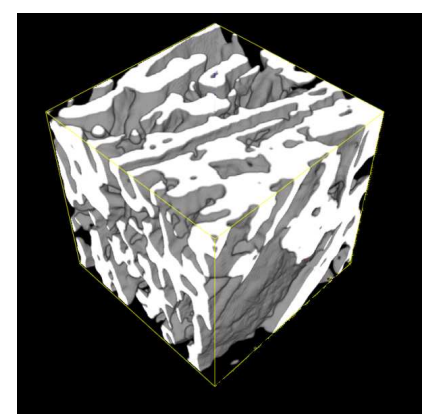

(a) $\widehat{f}_{\mathrm{SMI}}=0.50$

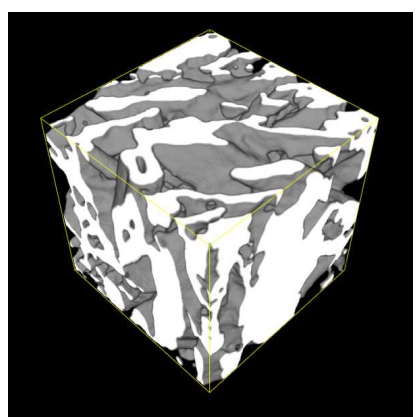

(b) $\widehat{f}_{\mathrm{SMI}}=-0.08$
Fig. 4. Visualisations of two $260^{3}$ pixel subvolumes of the reconstructed tomographic image of a recycling paper sample. Sample: Papiertechnische Stiftung (PTS), imaging: A. Rack, ESRF, ID-22.

\section{DISCUSSION}

Inspired by Hildebrand and Rüegsegger's structure model index we define in this paper a shape factor for macroscopically homogeneous random closed sets. This definition can be formulated without use of discretised realisations. Second, we derive a simple estimator for our SMI based on 3D image data. Contrary to the originally proposed estimator, ours does not require a surface meshing. Finally we prove that the SMI is of value for the comparison of similar microstructures while suffering from the same shortcomings as shape factors for compact sets.

Given the analogy between the SMI and the isoperimetric shape factors pointed out above, other shape factors could be explored, too. However, a direct replacement of V, S, and $\mathrm{M}$ in the formulae for the isoperimetric shape factors by the respective densities yields scale dependent quantities. In Hildebrand and Rüegsegger (1997a), two other shape factors, the structure volume exponent and the structure surface exponent, are suggested. These additionally depend on the thickness of the structure which is assumed to be constant throughout the sample. This assumption, however, is typically not met by real microstructures.

\section{ACKNOWLEDGEMENTS}

Joachim Ohser and Claudia Redenbach were supported by the $\mathrm{FH}^{3}$-programme of the German Federal Ministry of Education and Research under project grant 1711B06. Katja Schladitz was supported by the German Federal Ministry of Education and Research project 01 SF 0708-III-4a (FraunhoferCarnot cooperation).

\section{REFERENCES}

Chadœf J, Bacro J, Thébaud G, Labonne G (2008). Testing the boolean hypothesis in the non-convex case when a bounded grain can be assumed. Environmetrics 19:12336.

Davy P (1978). Stereology — A Statistical Viewpoint. Ph.D. thesis, Australian National University, Canberra.

Freitag J, Wilhelms F, Kipfstuhl S (2004). Microstructuredependent densification of polar firn derived from X-ray microtomography. J Glaciol 50:243-50.

Hildebrand T, Rüegsegger P (1997a). A new method for the model independent assessment of thickness in threedimensional images. J Microsc 185:67-75.

Hildebrand T, Rüegsegger P (1997b). Quantification of bone microarchitecture with the structure model index. Comput Methods Biomech Biomed Engin 1:15-23.

Hoffmann LM (2007a). Intersection densities of nonstationary Poisson processes of hypersurfaces. Adv Appl Probab 39:307-17.

Hoffmann LM (2007b). On weak stationarity and weak isotropy of processes of convex bodies and cylinders. Adv Appl Probab 39:864-82.

Lang C, Ohser J, Hilfer R (2001). On the analysis of spatial binary images. J Microsc 203:303-13.

Miles RE (1976). Estimating aggregate and overall characteristics from thick selections by transmission microscopy. J Microsc 107:227-33.

Ohser J, Nagel W, Schladitz K (2009). Miles formulae for Boolean models observed on lattices. Image Anal Stereol 29:77-92.

Ohser J, Schladitz K (2009). 3d Images of Materials Structures - Processing and Analysis. Heidelberg: Wiley.

Paganin D, Mayo SC, Gureyev TE, Miller PR, Wilkins SW (2002). Simultaneous phase and amplitude extraction from a single defocused image of a homogeneous object. J Microsc 206:33-40.

Schneider R (1993). Convex Bodies. The Brunn-Minkowski Theory. No. 44 in Encyclopedia of Mathematics and Its Application. Cambridge: Cambridge University Press.

Schneider R, Weil W (2008). Stochastic and Integral Geometry. Probability and Its Applications. Heidelberg: Springer.

Spiess M, Spodarev E (2009). Anisotropic Poisson processes of cylinders. Submitted. http://www. uni-ulm.de/fileadmin/website_uni_ulm/mawi.inst.110/ forschung/preprints/appc.pdf

Weitkamp T, Raven C, Snigirev AA (1999). Imaging and microtomography facility at the ESRF beamline ID 22. In: Bonse U, ed., Developments in X-Ray Tomography II. Proc SPIE 3772:311-7. 